\title{
Fabrication Techniques and RF Performances of Transmission Lines on Polymer Substrates
}

\author{
Jianqun Wang, Ying Cai, Thermpon Ativanichayaphong, Mu Chiao* and J.-C. Chiao \\ Department of Electrical Engineering, University of Texas at Arlington, USA \\ * Department of Mechanical Engineering, University of British Columbia, Canada \\ jxw4669@uta.edu
}

\begin{abstract}
The advantages of polymers have made them popular choices in many micro device applications. The benefits of low material and fabrication costs have been demonstrated in many micro-fluidic devices. The low conductivities and low dielectric constants of polymers provide potentials for high quality-factor RF MEMS applications. Nevertheless, the extension of using polymers for electronic components has not been well explored. In this work, we investigated the fabrication processes and RF performances of coplanar waveguide (CPW) transmission lines, with which many RF MEMS phase shifters, tuners, switches and interconnects are built, on polymer dielectric layers. In order to achieve optimum results, the CPW transmission lines were fabricated on benzocyclobutene (BCB), kapton and polyimide polymers. Our experimental results indicated very low insertion losses of $\mathrm{CPW}$ transmission lines with $\mathrm{BCB}$ as a dielectric layer and with kapton as substrate, and a moderate insertion loss with polyimide as a dielectric layer.
\end{abstract}

Keywords: Polymer, RF MEMS, Benzocyclobutene, Polyimide, Kapton, Coplanar Waveguide.

\section{INTRODUCTION}

The coplanar waveguide transmission lines have found many applications in RF MEMS utilizations such as RF phase shifters, tuners and switches. The CPW transmission lines have been widely utilized as interconnects in CMOS circuitries as well. The silicon substrates, on which most MEMS and IC devices were built, suffer from increasing insertion losses as the frequency increases. Many alternative approaches have been explored to achieve lower losses. Using quartz and high-resistivity silicon as substrates has been investigated, however the material costs are high. Adopting a layer of silicon dioxide [1], silicon nitride or oxidized porous silicon as an isolation layer may present a problem of thermal expansion mismatching between the silicon substrate and these dielectric layers [2]. Using the unoxidized porous silicon as a dielectric layer avoids the problem of thermal expansion mismatching [2], yet it limits many RF MEMS applications and is not suited to flexible processes due to the usage of hydrofluoric acid during the process of forming unoxidized porous silicon.

The ultra high resistivity [3] $\left(10^{19} \Omega \cdot \mathrm{cm}\right.$ or above) and low dielectric constant (2.65) of the benzocyclobutene polymer provide a very attractive option to the RF applications. Studies have shown a very low insertion loss [4] and high quality factor [5] in the millimeterwave frequencies with $\mathrm{BCB}$ as a dielectric layer. $\mathrm{BCB}$ does not have the ion-drifting problem with copper as a conductive layer without nitride as an isolation layer [6]. The low processing temperature $\left(250^{\circ} \mathrm{C}\right)$ is compatible with post CMOS processes. It is simple and cost-effective to control or vary BCB thickness through the spinon processes. Its resistance to some chemical agents, such as many acids, makes BCB compatible with most existing IC fabrication technologies. Its micromachining versatility is especially attractive for MEMS applications. Be it as a mechanical structure or sacrificial [7] layer, the microstructures can be fabricated either using photolithography, plasma etching [8], RIE etching, or hot embossing techniques.

The kapton polymer is a type of polyimide that has a low dielectric constant (3.4 3.5) and a high resistivity $\left(10^{17} \Omega \cdot \mathrm{cm}\right)$. The kapton is firm enough to be self supportive without the need of a backing substrate, thus any losses associated with the substrate could be avoided. It is resistant to many chemical agents, such as hydrochloric and sodium hydroxide, and many known organic solvents [9]. The polymer film maintains stable electrical and mechanical properties over a wide temperature range [9]. The unique properties of kapton make it an attractive material to RF MEMS applications.

Microelectronics: Design, Technology, and Packaging II, edited by Alex J. Hariz, Proc. of SPIE Vol. 6035, 60351M, (2006) · 0277-786X/06/\$15 · doi: 10.1117/12.637975 
Due to their excellent mechanical and electrical properties, we proposed to use BCB and kapton to build 3-D RF MEMS devices. Fig.1 shows our device structures. This paper investigates an optimal device design for high-frequency performance.

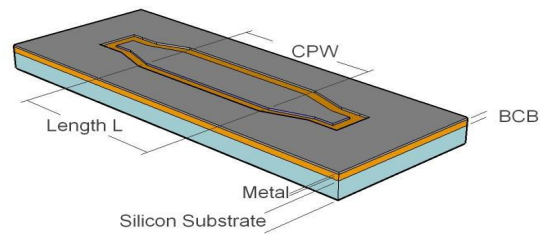

(a)

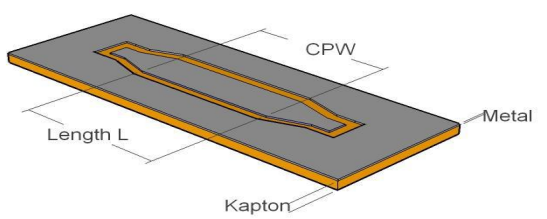

(c)

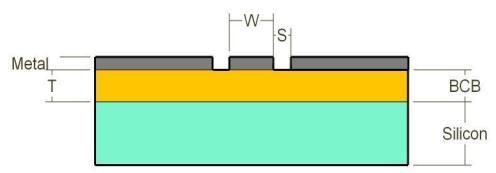

(b)

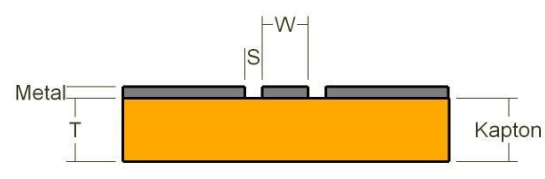

(d)

Figure 1. Coplanar waveguide transmission lines on polymer layers. (a) The conceptual structure of CPW lines with BCB as a dielectric layer and (b) the sideview. (c) The conceptual structure of CPW lines with kapton as a substrate and (b) the sideview.

\section{FABRICATION PROCESSES}

\subsection{Fabrication Process Using BCB as a Dielectric Layer}

Because of the popularity of low-resistivity silicon substrates and glass substrates, we built our CPW transmission lines on both materials, with the BCB polymer as a dielectric layer. The low-resistivity silicon substrate was cleaned with the RCA process and the glass substrate was cleaned with ultrasonic acetone bath to eliminate any contamination. A thin layer of surface promoter was applied to enhance the surface adhesion. The first BCB layer was spun on, followed by a soft-cure procedure at $210^{\circ} \mathrm{C}$ in an inert gas chamber for 40 minutes. In order to obtain a thick BCB layer, multiple spinning and curing processes were performed. The device was hard-cured at $250^{\circ} \mathrm{C}$ in the inert gas environment for 60 minutes. In our study, multiple thicknesses $(13,26$, and 36- $\mu \mathrm{m})$ of $\mathrm{BCB}$ were fabricated. A copper layer of 1.5- $\mu \mathrm{m}$ thickness was deposited on top of the $\mathrm{BCB}$ using thermal evaporation. The copper layer then was patterned with photolithography and wet etching processes.

The fabrication process using polyimide as a dielectric layer was similar to that of $\mathrm{BCB}$ except no surface promoter was needed and the final hard-cure temperature was $350^{\circ} \mathrm{C}$.

\subsection{Fabrication Process of Using Kapton as a Dielectric and Supportive Layer}

The manufacture process using kapton is relatively simple. The kapton film does not need a supporting substrate. The kapton film was first cut into square shape of 1 inch before copper thermal evaporation. After the copper evaporation, the copper layer was patterned with photolithography and wet etching processes.

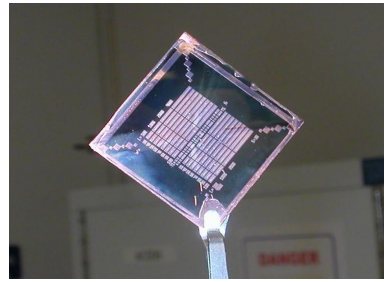

(a)

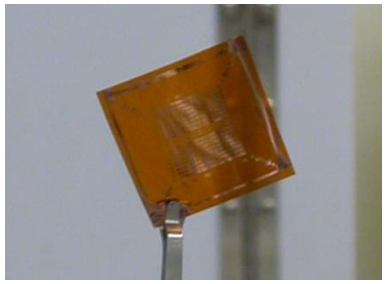

(b)

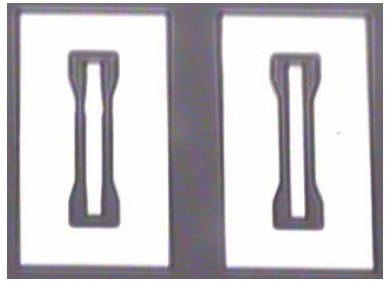

(c)

Figure 2. The fabricated coplanar waveguide transmission (CPW) lines. (a) The CPW lines with BCB as a dielectric layer and lowresistivity silicon as substrate. (b) The CPW lines built on a kapton polymer. (c) The CPW lines under the microscope. 


\section{RESULTS AND DISCUSSIONS}

The coplanar waveguide transmission line devices were characterized with an Agilent 8510 network analyzer and Cascade Microtech Infinity GSG-150 probes from DC to $26 \mathrm{GHz}$. The scattering parameter measurements were calibrated by the Cascade Microtech ISS-005-016 calibration kits. In this section, the effects of different elements on performances will be discussed.

\subsection{The Perfomances of Different Substrates}

Table 1 shows the device dimensions and their characteristic impedances for different substrate materials with BCB as a dielectric layer. Fig. 3 shows the measurement results of insertion loss and electric field simulations using finite element method (FEM).

\begin{tabular}{|c|c|c|c|c|c|c|}
\hline Substrate & Dielectric layer & $\mathrm{W}(\mu \mathrm{m})$ & $\mathrm{S}(\mu \mathrm{m})$ & $\mathrm{L}(\mathrm{mm})$ & $\mathrm{T}(\mu \mathrm{m})$ & $\mathrm{Z}_{\mathrm{O}}(\Omega)$ \\
\hline Glass & $\mathrm{BCB}$ & 400 & 20 & 4 & 36 & 52 \\
\hline Low-resistivity silicon & $\mathrm{BCB}$ & 70 & 20 & 4 & 36 & 54 \\
\hline
\end{tabular}

Table 1. The CPW dimensions and characteristic impedances for different substrate materials.

Fig. 3(a) and (b) show that the insertion loss is below $1.5 \mathrm{~dB} / \mathrm{cm}$ for the CPW lines built on BCB-glass and below $3.3 \mathrm{~dB} / \mathrm{cm}$ for the one built on BCB-silicon substrates. The BCB-silicon presenting a higher insertion loss can be explained as that the level of conductive dopants in low-resistivity silicon is much higher than in glass. Thus when the electromagnetic fields partially penetrate into the each substrate, as we can see from FEM field simulation in Fig. 3(c) and (d), the dopants in low-resistivity silicon generate a higher loss.

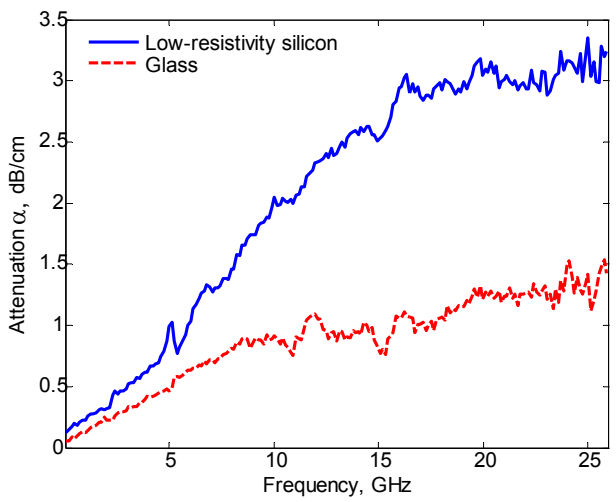

(a)

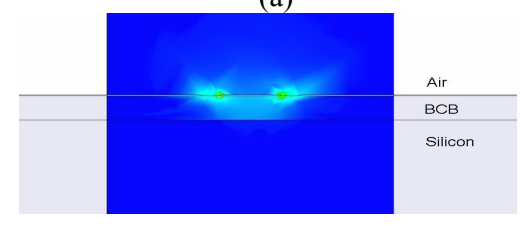

(c)

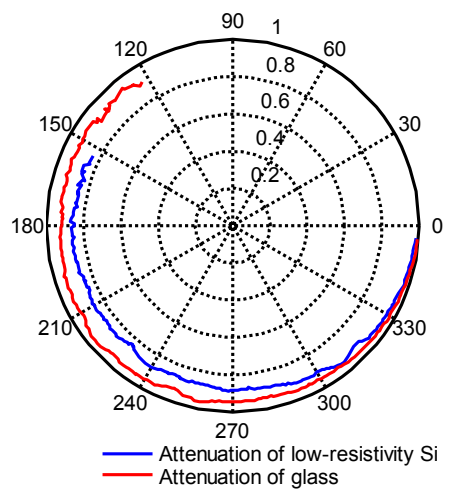

(b)

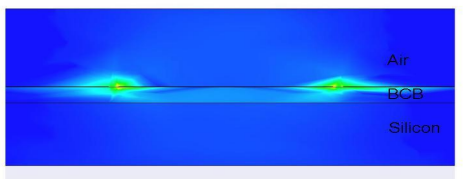

(d)

Figure 3. The performance comparison between silicon and glass substrates with $\mathrm{BCB}$ as a dielectric layer. (a) The magnitude comparison. (b) The Smith chart comparison. (c) The FEM field simulation of BCB-on-silicon. (d) The FEM field simulation of BCBon-glass.

\subsection{Improvement by Etching Polymer}

To reduce field coupling in the dielectric, one could reduce the dielectric losses. Using the reactive ion etching process (RIE), we etched away the polymer between the signal and ground metals. With the CPW line metal layer serving as a hard mask, the air slots were achieved by $2: 1$ oxygen to tetrafluoromethane ratio. Table 2 shows the CPW line dimensions used in etching experiments. Fig. 4 shows the measurement and FEM field simulation results. 


\begin{tabular}{|c|c|c|c|c|c|}
\hline Polymer & Substrate & $\mathrm{W}(\mu \mathrm{m})$ & $\mathrm{S}(\mu \mathrm{m})$ & $\mathrm{L}(\mathrm{mm})$ & $\mathrm{T}(\mu \mathrm{m})$ \\
\hline BCB & Low-resistivity silicon & 60 & 20 & 2 & 36 \\
\hline BCB & Glass & 400 & 20 & 4 & 36 \\
\hline
\end{tabular}

Table 2. The CPW dimensions for different substrate materials.

After etching the BCB polymer and a 13- $\mu \mathrm{m}$ depth into the silicon substrate between the signal and ground metals, the insertion loss of CPW lines on BCB-silicon was improved by $2 \mathrm{~dB} / \mathrm{cm}$ at the high frequency range (Fig. 4(a)). This is, firstly, due to the air between signal and ground metals presents less losses than silicon [10] and polymer. Secondly, after etching the $\mathrm{BCB}$ and silicon, fewer fields couple and penetrate into the silicon substrate, as shown in the field simulation in Fig. 4(c). Thus a lower attenuation was achieved. On the other hand, on the BCB-glass device the slot width $(20-\mu \mathrm{m})$ is much smaller comparing to the signal conductor width $(400-\mu \mathrm{m})$ (Fig. 4(d)), thus even with the air serving as a better dielectric material, the improvement was insignificant (Fig. 4(b)) since the field concentration and penetration does not change significantly. Noted that the RIE process only removed BCB and silicon, but not glass.

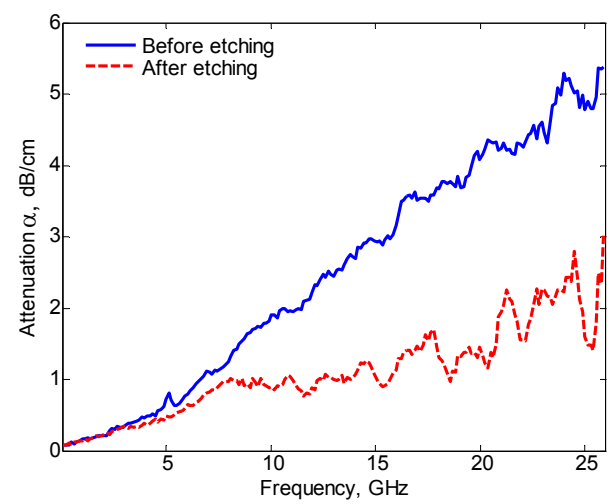

(a)

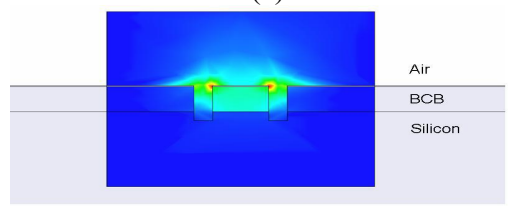

(c)

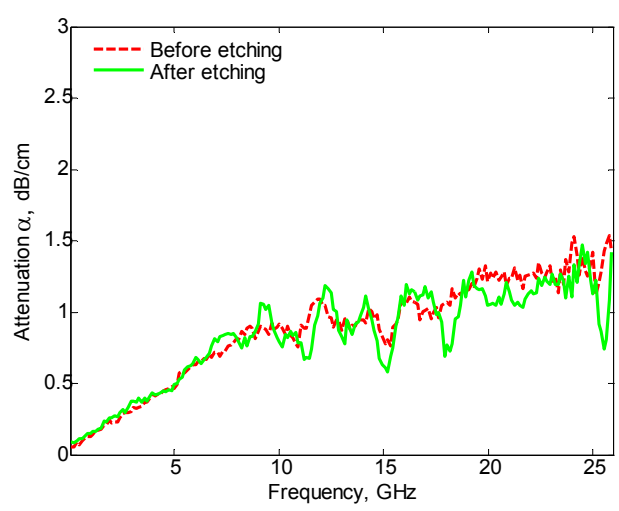

(b)

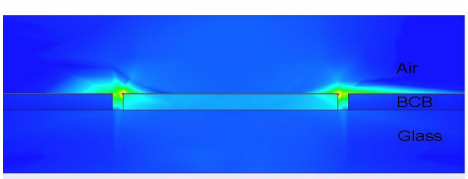

(d)

Figure 4. The performance comparison before and after etching. (a) The attenuation before and after etching BCB and a 13- $\mu \mathrm{m}$ depth into the silicon substrate. (b) The attenuation before and after etching BCB, but not into the glass substrate. (c) The FEM field simulation after etching BCB and the silicon substrate for a 13- $\mu \mathrm{m}$ depth. (d) The FEM field simulation after etching BCB, but not into the glass substrate.

\subsection{The Perfomances of Different Polymers}

\subsubsection{Comparison between BCB and kapton}

The CPW lines built on kapon don't need silicon or glass as substrates, thus we expect a reduction of insertion losses associated with the substrates. The CPW lines were designed to have matched characteristic impedances. The dimensions are shown in the Table 3. Fig. 5 shows the measurement results and electric field simulations.

\begin{tabular}{|c|c|c|c|c|c|c|}
\hline Polymer & Substrate Material & $\mathrm{W}(\mu \mathrm{m})$ & $\mathrm{S}(\mu \mathrm{m})$ & $\mathrm{L}(\mathrm{mm})$ & $\mathrm{T}(\mu \mathrm{m})$ & $\mathrm{Z}_{\mathrm{O}}(\Omega)$ \\
\hline BCB & Low-resistivity silicon & 70 & 20 & 4 & 36 & 54 \\
\hline Kapton & No substrate & 100 & 10 & 4 & 127 & 49 \\
\hline
\end{tabular}

Table 3. The CPW dimensions and characteristic impedances for different polymer materials. 


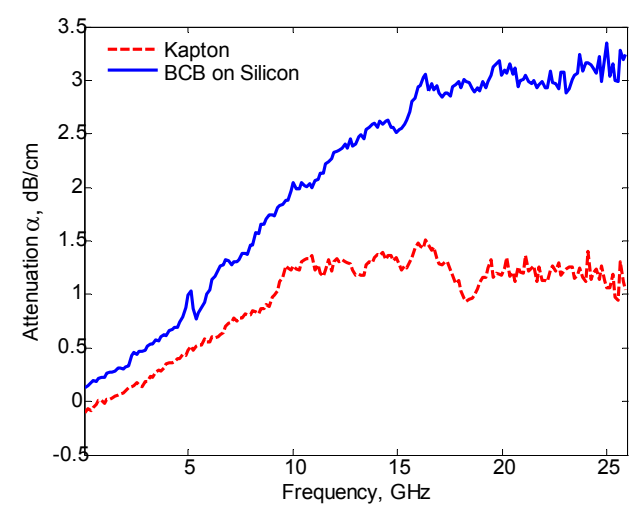

(a)

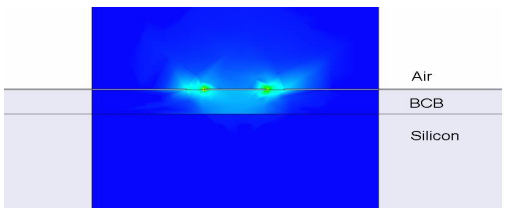

(b)

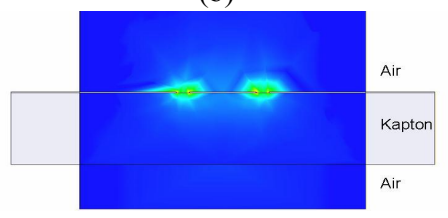

(c)

Figure 5. The performance comparison between BCB and kapton. (a) The measurement results. (b) The field simulation of a CPW line on the BCB-silicon. (c) The field simulation of a CPW line on the kapton.

The kapton presents an attenuation below $1.5 \mathrm{~dB} / \mathrm{cm}$ up to $26 \mathrm{GHz}$. The attenuation for a $36-\mu \mathrm{m} \mathrm{BCB}$ layer on silicon is below $3.3 \mathrm{~dB} / \mathrm{cm}$. The lower attenuation of the CPW line on kapton is, primarily, due to the lower losses for the coupled fields into the substrate as shown in the field simulation in the Fig. 5(c). The CPW line on kapton does not have a low-resistivity silicon as a substrate. Therefore, it eliminates any loss associated by the low-resistivity silicon. For $\mathrm{CPW}$ lines on $\mathrm{BCB}$-silicon, as the electromagnetic fields penetrate through $\mathrm{BCB}$ into the substrate (Fig. 5(b)), the dopants induce a higher loss than polymers.

\subsubsection{Comparison between $B C B$ and polyimide}

With low-resistivity silicon as substrates, polyimide was also used as a dielectric layer to build CPW transmission lines to compare with the BCB polymer. Table 4 shows the device dimensions. Fig. 6 shows the measurement and FEM simulation results.

\begin{tabular}{|c|c|c|c|c|c|}
\hline Polymer & Substrate & $\mathrm{W}(\mu \mathrm{m})$ & $\mathrm{S}(\mu \mathrm{m})$ & $\mathrm{L}(\mathrm{mm})$ & $\mathrm{T}(\mu \mathrm{m})$ \\
\hline BCB & Low-resistivity silicon & 60 & 20 & 2 & 13 \\
\hline Polyimide & Low-resistivity silicon & 60 & 20 & 2 & 16 \\
\hline
\end{tabular}

Table 4. The CPW dimensions for different polymer materials.

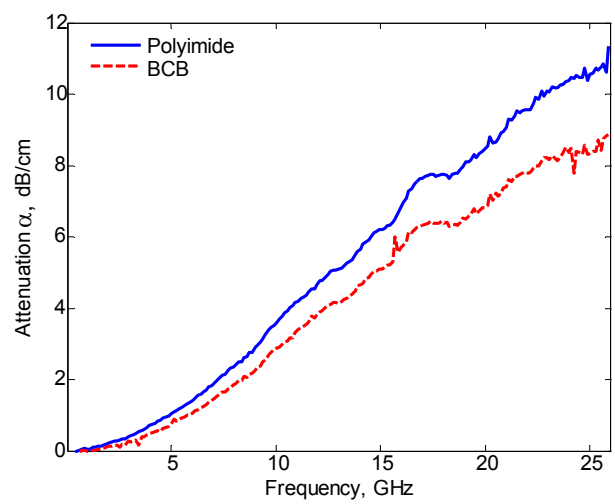

(a)

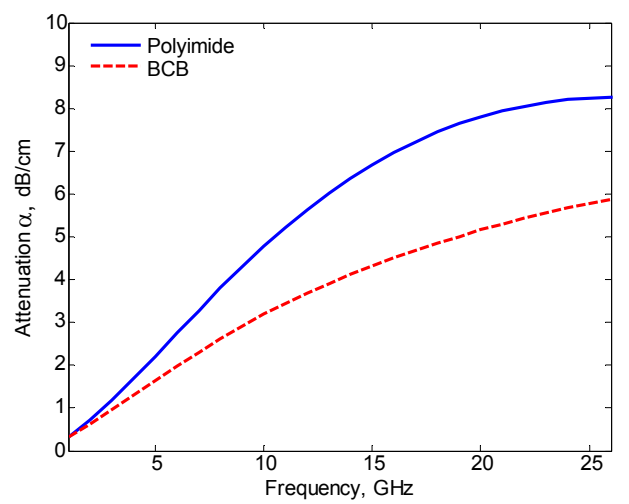

(b)

Figure 6. The performance comparison between polyimide and BCB. (a) The measurement results. (b) The simulation results.

The thicker 16- $\mu \mathrm{m}$ polyimide layer suffers slightly higher insertion loss than a thinner 13- $\mu \mathrm{m}$ BCB layer (Fig. 6(a)). This is due to the inherent electrical properties. The BCB has a lower dielectric constant (2.65) and a higher resistivity $\left(10^{19} \Omega \cdot \mathrm{cm}\right)$ while the polyimide has a dielectric constant of 2.9 and a resistivity of $10^{17} \Omega \cdot \mathrm{cm}$. Thus the electromagnetic 
fields encounter less losses within the BCB polymer than polyimide. Simulation results in Fig. 6(b) confirm our experimental results.

\subsection{The Performances of Different Polymer Thicknesses}

We designed and fabricated CPW lines on different thickness polymers $(13,26$ and $36-\mu \mathrm{m})$. Table 5 shows the device dimensions. Fig. 7 shows the measurement results and FEM field simulation results.

\begin{tabular}{|c|c|c|c|c|c|}
\hline Polymer & Substrate & $\mathrm{W}(\mu \mathrm{m})$ & $\mathrm{S}(\mu \mathrm{m})$ & $\mathrm{L}(\mathrm{mm})$ & $\mathrm{T}(\mu \mathrm{m})$ \\
\hline BCB & Low resistivity silicon & 60 & 20 & 1 & 13 \\
\hline BCB & Low resistivity silicon & 60 & 20 & 1 & 26 \\
\hline BCB & Low resistivity silicon & 60 & 20 & 1 & 36 \\
\hline
\end{tabular}

Table 5. The CPW dimensions for different polymer thicknesses.

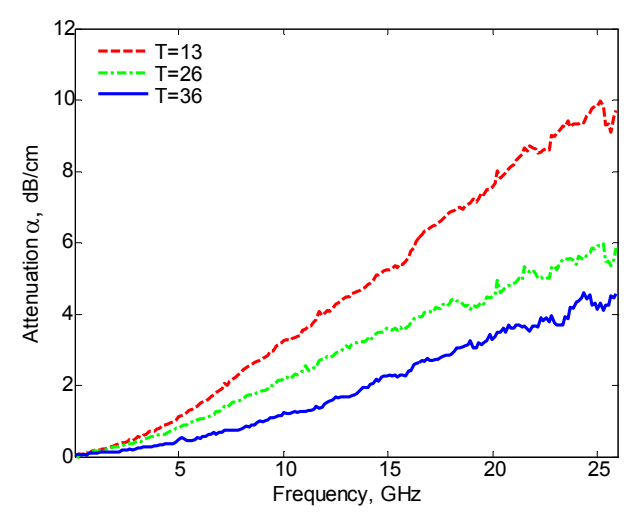

(a)

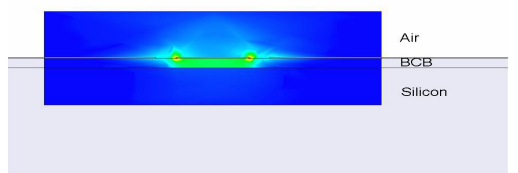

(b)

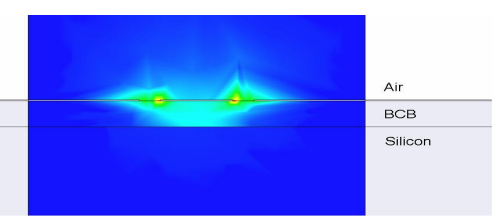

(c)

Figure 7. The performance comparison between different polymer thicknesses. (a) The measurement results. (b) The FEM field simulation of a 13- $\mu \mathrm{m}$ thick BCB layer. (b) The FEM field simulation of a 36- $\mu \mathrm{m}$ thick BCB layer.

A thicker polymer confines the electromagnetic fields better than the thin one does (Fig. 7(b) and (c)), hence less electromagnetic fields would be leaked into the loss-inducing silicon substrate. The measurement results (Fig. 7(a)) indicate that the thickest $\mathrm{BCB}$ polymer presents a lowest loss. At $10 \mathrm{GHz}$, the insertion loss of 36- $\mu \mathrm{m}$ thick $\mathrm{BCB}$ is below $1.5 \mathrm{~dB} / \mathrm{cm}$, meanwhile the insertion loss of $13-\mu \mathrm{m}$ thick BCB is below $3.5 \mathrm{~dB} / \mathrm{cm}$. It has been reported a $4 \mathrm{~dB} / \mathrm{cm}$ insertion loss for a 10- $\mu \mathrm{m}$ BCB polymer [4], which is consistent with our experimental trends.

\subsection{The Impacts of Device Dimensions}

\subsubsection{The impacts of CPW widths}

To investigate the impacts of the center metal signal line, we fixed the signal-ground gap, S, and varied signal line widths on a fixed-length CPW lines. Table 6 shows the device dimensions and their characteristic impedances. Fig. 8 shows the measurement results and FEM field simulation results.

\begin{tabular}{|c|c|c|c|c|c|c|}
\hline Polymer & Substrate & $\mathrm{W}(\mu \mathrm{m})$ & $\mathrm{S}(\mu \mathrm{m})$ & $\mathrm{L}(\mathrm{mm})$ & $\mathrm{T}(\mu \mathrm{m})$ & $\mathrm{Z}_{\mathrm{O}}(\Omega)$ \\
\hline $\mathrm{BCB}$ & Low-resistivity silicon & 30 & 20 & 1 & 36 & 82 \\
\hline BCB & Low-resistivity silicon & 70 & 20 & 1 & 36 & 54 \\
\hline BCB & Low-resistivity silicon & 80 & 20 & 1 & 36 & 51 \\
\hline BCB & Low-resistitvity silicon & 100 & 20 & 1 & 36 & 44 \\
\hline
\end{tabular}

Table 6. The CPW dimensions and characteristic impedances.

The CPW line with a $30-\mu \mathrm{m}$ width presents a biggest attenuation due to its largely unmatched characteristic impedance. Intuitively, the matched CPW line with a $80-\mu \mathrm{m}$ signal width is expected to have a lower loss than the 100$\mu \mathrm{m}$ wide CPW line. The measurement results (Fig. 8(a)), however, indicated that a wider center conductor provide a less insertion loss. This is due to the wider center conductor presents a less concentrated field, thus the less skin-depth effect 
results in a lower attenuation. The field simulations in Fig. 8(b), (c) and (d) show a wider conductor has a less field concentration.

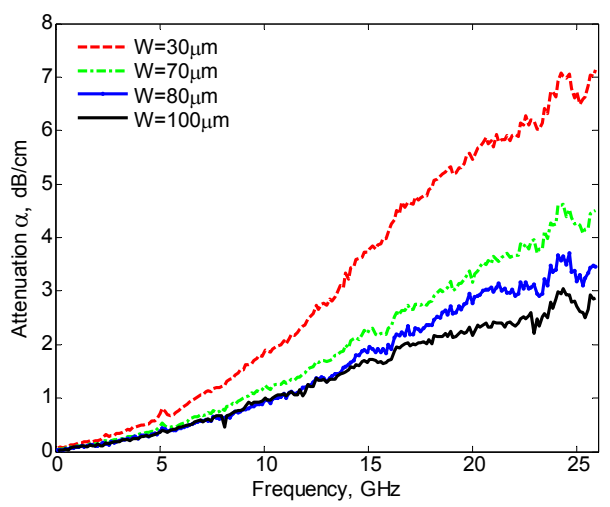

(a)

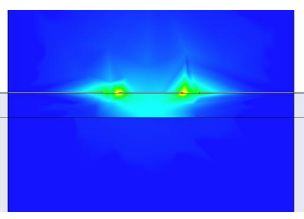

(b)

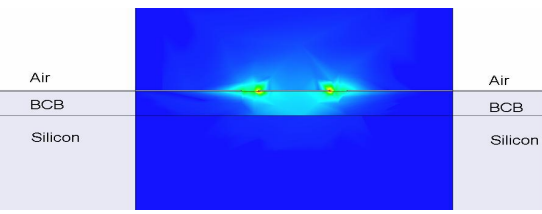

(c)

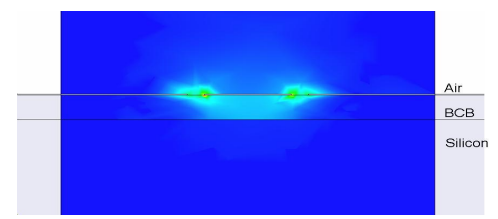

(d)

Figure 8. The performance comparison between different signal conductor widths. (a) The measurement results. (b) The FEM field simulation for a $70-\mu \mathrm{m}$ width center conductor. (c) The FEM field simulation for a $80-\mu \mathrm{m}$ width center conductor. (d) The FEM field simulation for a $100-\mu \mathrm{m}$ width center conductor.

\subsubsection{The impacts of CPW lengths}

We varied CPW line lengths and fixed signal width, $W$, and gap, $S$, to verify our measurements. Table 7 shows the dimensions and their characteristic impedances. Fig. 9 shows the measurement and simulation results for the total loss. The curves show a proportional trend with the length. This measurement is to establish the baseline for measurement accuracy. The measurement trends indicate the unit loss is about $0.6 \mathrm{~dB} / \mathrm{mm}$ at $20 \mathrm{GHz}$.

\begin{tabular}{|c|c|c|c|c|c|c|}
\hline Polymer & Substrate material & $\mathrm{L}(\mathrm{mm})$ & $\mathrm{W}(\mu \mathrm{m})$ & $\mathrm{S}(\mu \mathrm{m})$ & $\mathrm{T}(\mu \mathrm{m})$ & $\mathrm{Z}_{\mathrm{O}}(\Omega)$ \\
\hline BCB & Low-resistivity silicon & 0.5 & 70 & 20 & 36 & 54 \\
\hline BCB & Low-resistivity silicon & 1 & 70 & 20 & 36 & 54 \\
\hline BCB & Low-resistivity silicon & 2 & 70 & 20 & 36 & 54 \\
\hline
\end{tabular}

Table 7. The CPW dimensions and characteristic impedances.

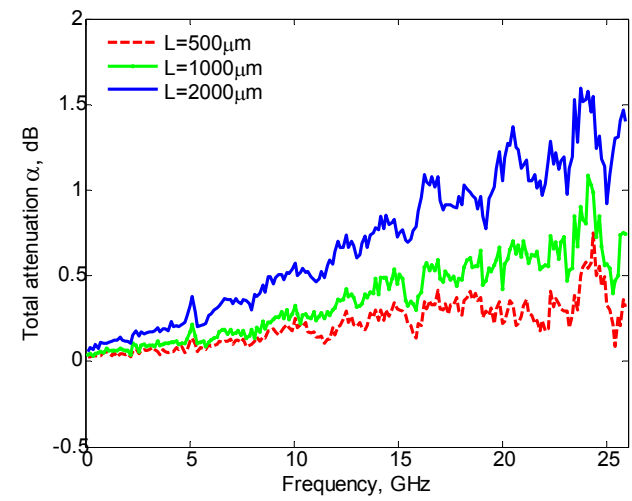

(a)

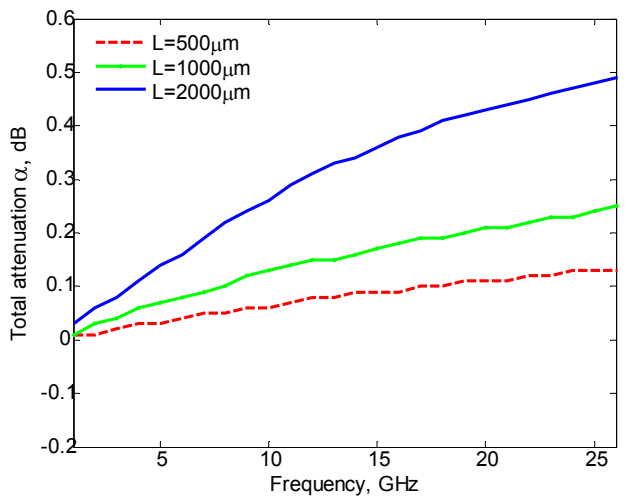

(b)

Figure 9. The insertion losses of CPW lines with different lengths. (a) The measurement results. (b) The FEM simulation results.

\subsubsection{The impacts of the gap sizes}

To show the impacts of the gap size, we fixed the width, $W$, and vary the gap, $S$, on fixed-length CPW lines. Table 8 shows the dimensions. Fig. 10 shows the measurement results and FEM field simulations. 


\begin{tabular}{|c|c|c|c|c|c|}
\hline Polymer & Substrate material & $\mathrm{S}(\mu \mathrm{m})$ & $\mathrm{W}(\mu \mathrm{m})$ & $\mathrm{L}(\mathrm{mm})$ & $\mathrm{T}(\mu \mathrm{m})$ \\
\hline BCB & Low-resistivity silicon & 10 & 70 & 1 & 36 \\
\hline BCB & Low-resistivity silicon & 20 & 70 & 1 & 36 \\
\hline BCB & Low-resistivity silicon & 40 & 70 & 1 & 36 \\
\hline
\end{tabular}

Table 8. The CPW dimensions.

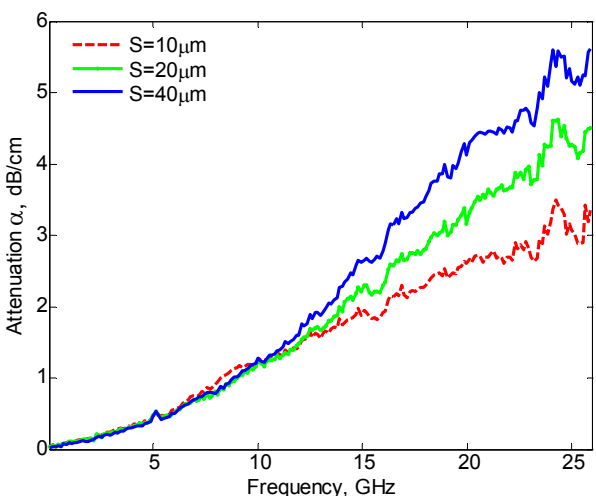

(a)

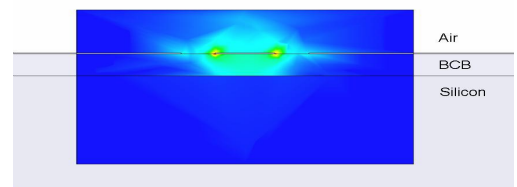

(b)

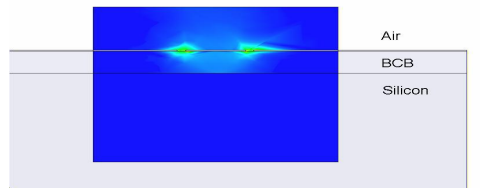

(c)

Figure 10. The insertion losses of CPW lines with different signal-ground gaps. (a) The measurement results. (b) The field simulation for a $40-\mu \mathrm{m}$ gap. (c) The field simulation for a $10-\mu \mathrm{m}$ gap.

Our experimental results show (Fig. 10(a)) the CPW line with widest gap suffers from the highest loss. This is possibly due to that a wider gap results in more coupled fields into the substrate, as indicated in the FEM field simulation in Fig. 10(b) and Fig. 10(c), thus the attenuation induced by the dopants of substrates is higher [11].

\subsection{The Impacts of Matching Circuits}

The probes used have CPW GSG contacts with a spacing of $150-\mu \mathrm{m}$ and width of $50-\mu$ m. Smooth impedance transitions were designed to accommodate different CPW line dimensions. Table 9 shows the matching circuit dimensions and their characteristic impedances. Fig. 11 shows the linear transitions of matching circuits between probe ends and the CPW lines. The insertion losses induced by such matching circuits are estimated to be smaller than 0.15 $\mathrm{dB} / \mathrm{cm}[12]$.

\begin{tabular}{|c|c|c|c|c|c|c|}
\hline Polymer & Substrate & $\mathrm{W}(\mu \mathrm{m})$ & $\mathrm{L}(\mu \mathrm{m})$ & $\mathrm{S}(\mu \mathrm{m})$ & $\mathrm{T}(\mu \mathrm{m})$ & $\mathrm{Z}_{\mathrm{O}}(\Omega)$ \\
\hline $\mathrm{BCB}$ & Glass & 140 & 100 & 10 & 30 & 50.1 \\
\hline BCB & Silicon & 80 & 100 & 50 & 30 & 50.8 \\
\hline
\end{tabular}

Table 9. The taper dimensions and characteristic impedances.

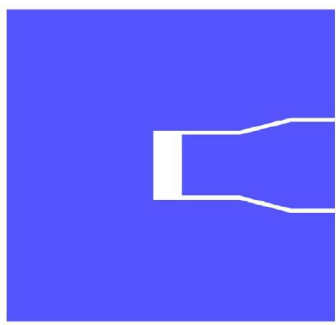

(a)

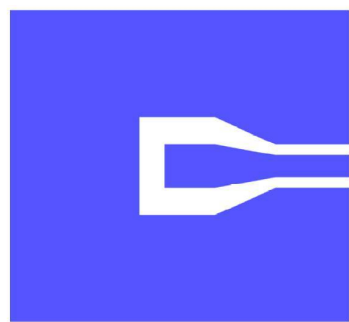

(b)

Figure 11. Taper matching circuits. (a) The matching circuits for CPW lines on BCB-glass. (b) The matching circuits for CPW lines on BCB-silicon. 


\section{CONCLUSIONS}

In this paper, we investigated the fabrication techniques and RF performances of $\mathrm{CPW}$ transmission lines on different organic dielectric materials and substrate materials. The effects of device dimensions were studied as well. Our measurement and simulation results indicated that the BCB and kapton polymer present very low insertion losses. The fabrication techniques are simple, low-cost and compatible with existing CMOS processes.

\section{ACKNOWLEDGMENTS}

This research is sponsored by the National Science Foundation DMI \#0428884.

The authors would like to express their gratitude to Drs. Alan Davis and Ronald Carter for their help on characterization supports, Drs. Nasir Basit, Vance Ley and Dan Popa for the cleanroom fabrication assistances, and Mr. Peter Lun-Chen Hsu for his help on device testing.

\section{REFERENCES}

1. Bendik Kleveland, Thomas H. Lee and S. Simon Wong, "50-GHz interconnect design in standard silicon technology," International Microwave Theory and Techniques Symposium, Volume 3, pp.1913-1916, 1998.

2. Han-Su Kim, Ya-Hong Xie, Marc Devincentis, Tatsuo Itoh, and Keith Jenkins, "Unoxidized porous Si as an isolation material for mixed-signal integrated circuit applications," Journal of Applied Physics, Volume 93, Issue 7, pp. 4226-4231, 2003.

3. DOW Processing Procedures For CYCLOTENE 4000 Series Resin.

4. K. Grenier, V. Lubecke, F. Bouchriha, L. Rabbia. D. Dubuc, P. Pons, and R. Plana, "Polymer in RF and milimeterwave applications," Proc. of SPIE, Volume 5116, pp. 502-513, 2003.

5. Xiao Huo, Kevin J. Chen, and Philip C. H. Chan, "Silicon-based high-Q inductors incorporating electroplated copper and low-K BCB dielectric", IEEE Electron Device Letters, Volume 23, Issure 9, pp. 520-522, 2002.

6. A.L.S. Loke, J.T. Wetzel, Changsup Ryu, Won-Jun Lee, and S.S. Wong, "Copper drift in low-K polymer dielectrics for ULSI metallization,” 1998 Symposium of VLSI Technology, pp.26 -27, June 1998.

7. Zhimin Yao and Noel C. MacDonald, "Single crystal silicon supported thin film micromirrors for optical applications," Optical Engineering, Volume 36, Issue 5, pp. 1408-1413, May 1997.

8. Sang Won Park, Kabseog Kim, Jeong-Bong Lee, and Wendel Davis, "Plastic-based pattern transfer process for RF MEMS passives," IEEE 2002 Emerging Telecommunication Technologies Conference, Richardson, TX, September 2002.

9. "Kapton Summary of Properties", DoPont.

10. J. Chuang, S.M. El-Ghazaly, D.K. Schroder, Y.H. Zhang, and G.N. Maracas, A.C.Reyes, "Air-gap transmission lines for OEICs and MMICs using glass substrates," IEEE MTT-S International Microwave Symposium Digest, Volume 1, pp.265 - 268, 1997

11. L.L.W. Leung, Wai-Cheong Hon and K. J. Chen, "Low-loss coplanar waveguides interconnects on low-resistivity silicon substrate," IEEE Transactions Components and Packaging Technologies, Volume 27, Issue 3, pp.507 512, Sept. 2004.

12. Janusz Grzyb and Gerhard Tröster, "Characteristic impedance deembeding of printed lines with probe-tip calibrations," Proc. 32nd European Microwave Conference, Milan, Italy, September 23-26, 2002. 